\title{
Astronomy and Development in Southern Africa
}

\author{
Patricia A. Whitelock \\ South African Astronomical Observatory \\ PO Box 9, Observatory, 7935, South Africa \\ and Department of Astronomy, University of Cape Town \\ Rondebosch, 7701, South Africa \\ E-mail: pawesaao.ac.za
}

\begin{abstract}
Africa has the potential to make major contributions to international astronomy. The South African government has identified astronomy as an area in which the country has a strategic advantage, by virtue of its geographical position, good climate and well-established astronomical community. They have therefore invested heavily in projects such as the Southern African Large Telescope (the largest single optical telescope in the Southern Hemisphere) and the Karoo Array Telescope (MeerKAT, as a demonstrator for the Square Kilometre Array). The investment is intended not only to facilitate an African contribution to astronomy, but also to use astronomy as a tool for national development. It is vital that all involved in this appreciate that scientific success is a prerequisite for any meaningful contribution to the development agenda. The success or failure of these endeavours will have far reaching consequences for astronomy and for Africa. Which way it goes will depend as much on the international astronomy community as it will on African politics.
\end{abstract}

Accelerating the Rate of Astronomical Discovery - sps5

Rio de Janeiro, Brazil

August 11-14 2009 


\section{Introduction}

Although this talk was scheduled in the part of the meeting entitled "discrimination", it is not so much about discrimination as about opportunity - opportunity for people who have never previously been exposed to astronomy in any significant way to get involved in astronomical activity. The government of South Africa has identified astronomy as an area in which the country has a strategic advantage by virtue of its geographical position, good climate and well-established astronomy community. They have therefore invested heavily in projects such as the Southern African Large Telescope (SALT) and the Karoo Array Telescope (MeerKAT as a demonstrator for the Square Kilometre Array). This investment is intended not only to facilitate an African contribution to astronomy, but also to use astronomy as a tool for national development. In this paper I describe the astronomy facilities, the major significant pieces of legislation and the human development programmes currently underway. The discussion is somewhat biased towards optical astronomy, as that is the area with which I am most familiar, but I hope that I also give a sense of the very exciting developments going on at other wavelengths.

\section{Optical Astronomy}

The South African Astronomical Observatory (SAAO) ${ }^{1}$ is the main facility for optical astronomy. It is financed by the government through the Department of Science and Technology (DST) as a National Facility of the National Research Foundation (NRF). SAAO is headquartered in Cape Town, while its telescopes are located at Sutherland in the Northern Cape, about $360 \mathrm{~km}$ by road from Cape Town at an altitude of $1800 \mathrm{~m}$. The main instrument at this site is the Southern African Large Telescope (SALT) ${ }^{2}$, which, in late 2009, was nearing the end of its commissioning period. This is a $10 \mathrm{~m}$-class telescope, with significant design changes from its prototype, the Hobby-Eberly Telescope in Texas, USA. SALT is operated by SAAO on behalf of the international consortium that owns it; this includes partners from USA, Poland, India, Germany, UK New Zealand, and South Africa. The project originated in South Africa, which owns the largest single share.

SALT's current instrumentation includes SALTICAM, a science/acquisition camera with an $8^{\prime}$ diameter field of view and a variety of filters, and the Robert Stobie Spectrograph (RRS), which offers medium resolution multi-object spectroscopy over the full field as well as spectropolarimetry, and Fabry-Perot imaging over a narrower field of view. RRS is optimized for use in the ultraviolet, although a near-infrared arm is under construction as a second-generation instrument. A high-resolution spectrograph is also under construction.

\footnotetext{
${ }^{1}$ www.saao.ac.za

${ }^{2}$ www.salt.ac.za
} 


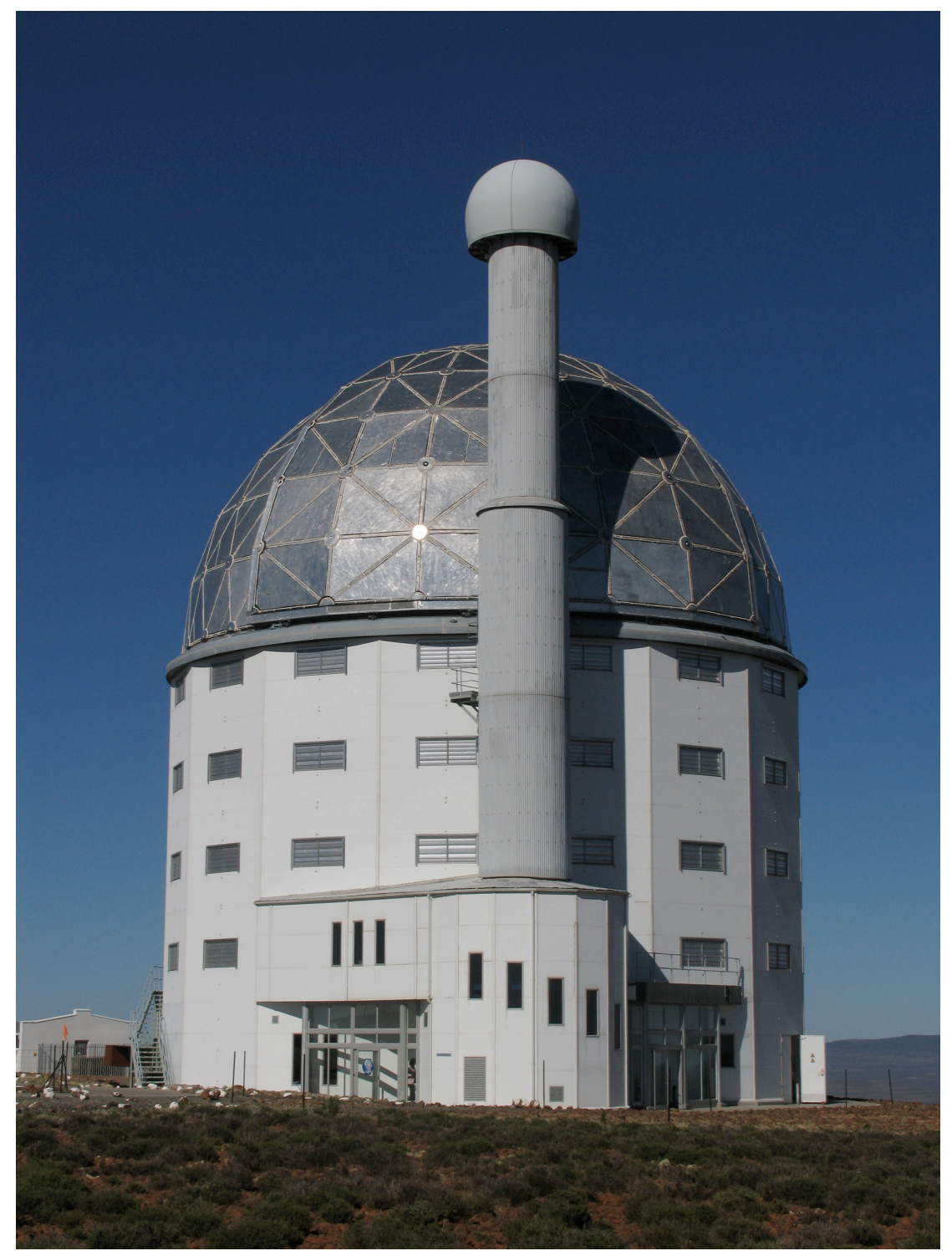

Figure 1The SALT telescope dome at Sutherland in South Africa

SALT's low cost construction, using a spherical primary and a fixed altitude supporting structure makes it a test-bed for technology that could be used on the next generation of extremely large telescopes [1].

In addition to SALT, SAAO is home to numerous small telescopes, including general user telescopes with apertures ranging from 0.5 to $1.9 \mathrm{~m}$ that are owned by South Africa. These are used by the local community for a variety of purposes, including student training. They are also available for international users and time is preferentially allocated to SALT partners, particularly for work in support of SALT. 
There are also a number of other telescopes, many of them automated, that are run on behalf of, or jointly with, international partners from, e.g. Korea, Germany, the UK and USA. The largest of these is a $1.4 \mathrm{~m}$ Infrared Survey Facility run jointly with Japan. These various facilities have been attracted to Sutherland by the location and/or the available high-tech infrastructure. The location is obviously attractive because of the unique latitude and longitude and excellent climate. Less obvious is the high seismic stability which makes Sutherland the ideal location for certain non-astronomical experiments, such as a Geodynamic Observatory run by GeoForschungsZentrum from Potsdam, Germany and a seismometer operated as part of the "International Deployment of Accelerometers" (IDA) project run from the University of California, USA.

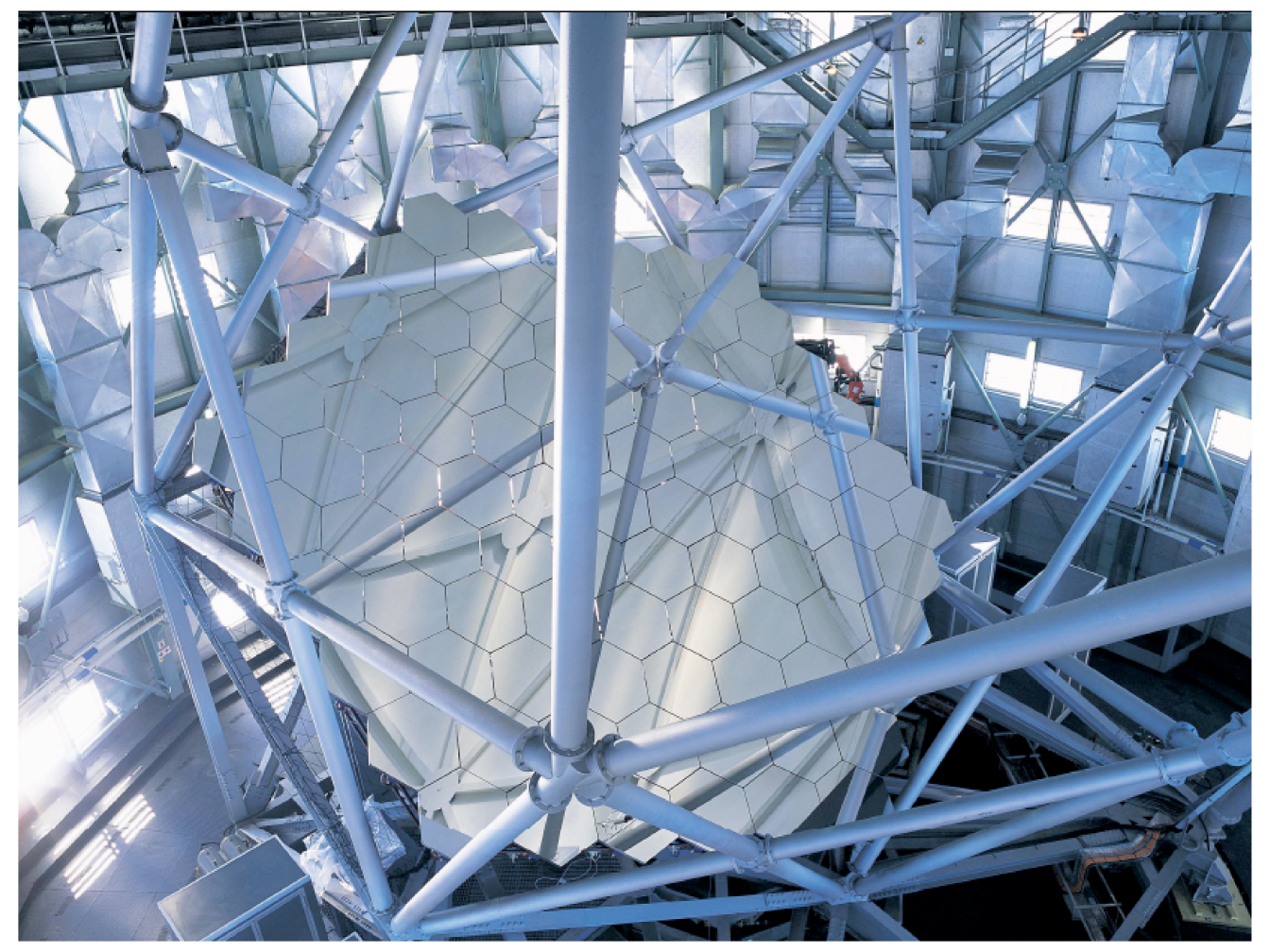

Figure 2The SALT primary mirror comprises 91 identical hexagonal segments.

The developments at Sutherland can be seen as part of a broader plan to make South Africa an attractive destination for a variety of astronomical projects. While most projects involve potential collaborations between South African and international astronomers, the small size of the local community has limited the exploitation of the opportunities available. 


\section{Radio Astronomy}

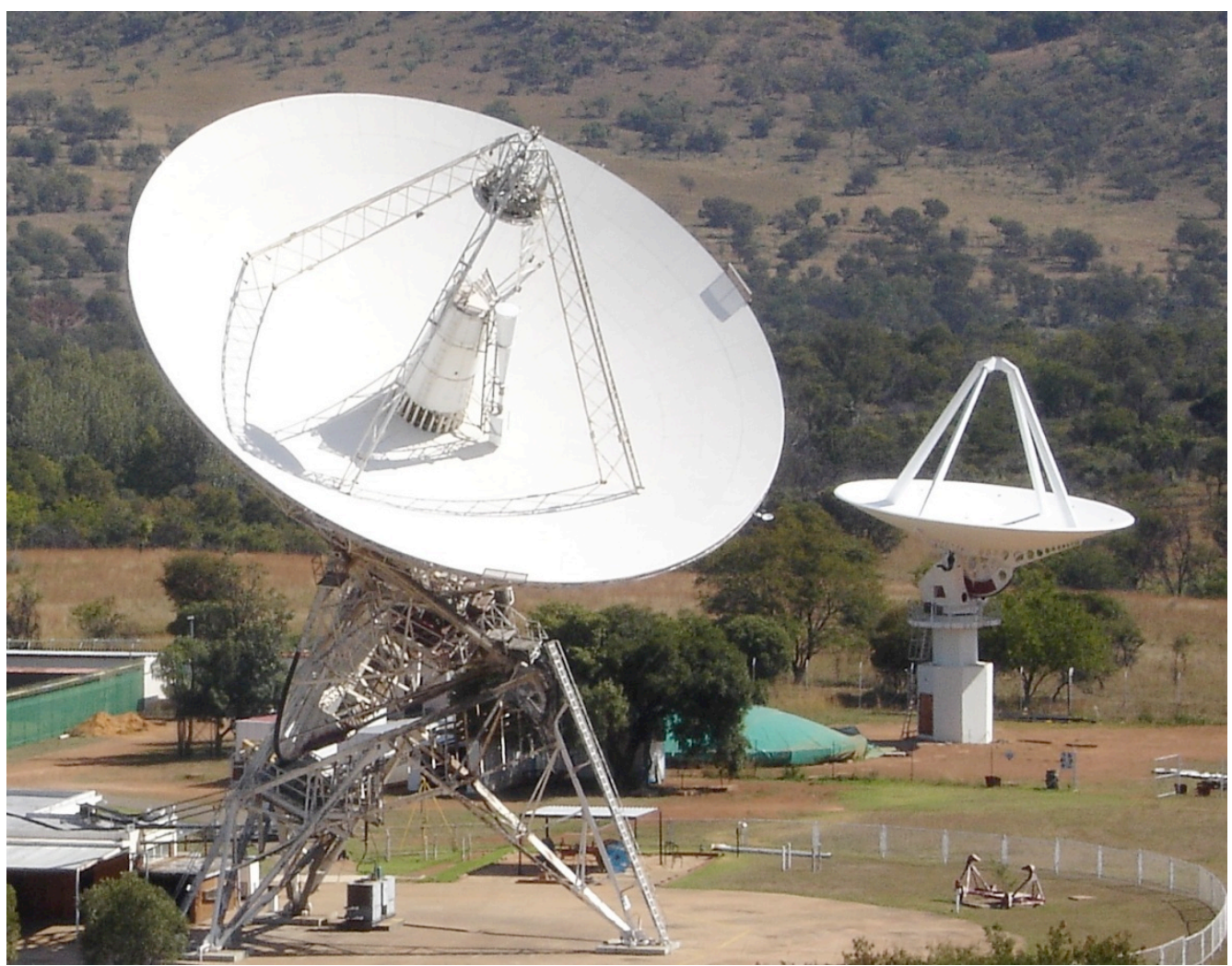

Figure 3The Hartebeesthoek $26 \mathrm{~m}$ antenna, with the $15 \mathrm{~m}$ eXperimental Development Model (XDM) testbed prototype for KAT (credit: Mike Gaylard).

The Hartebeesthoek Radio Observatory (HartRAO) ${ }^{3}$ is the National Facility for radio astronomy and is financed and administered in the same way as SAAO. It has operated a 26m radio telescope since 1974 (see Fig. 3). The antenna was built by NASA in 1961, originally as part of their deep space network for tracking spacecraft beyond Earth orbit. Because of its unique location on the southern tectonic plate it has played an important role in the world's VLBI networks for both radio astronomy and geodesy. The antenna has also been used extensively for monitoring the temporal variations of cosmic masers and selected pulsars. In the period 2000 to 2004 the surface of the antenna was upgraded to enable it to operate at frequencies above $15 \mathrm{GHz}$. Unfortunately, in late 2008 the antenna suffered a failure of the major bearing on the polar drive shaft and this is now in the process of being repaired.

During 2007 a 15m antenna of innovative design (composite one piece reflector) was constructed at Hartebeesthoek as a test-bed prototype for the Karoo Array Telescope (Fig. 3).

\footnotetext{
${ }^{3}$ www.hartrao.ac.za
} 
South Africa is on the short list, together with Australia, to host the Square Kilometre Array (SKA) radio telescope. The Karoo Array Telescope ${ }^{4}$ will be used to test technology leading up to the development of the SKA and will be a very powerful telescope in its own right. The first seven antennae for KAT are under construction in the Karoo (Fig. 4) and should be operational towards the end of 2009. The full array, of at least 80 dishes, is scheduled for 2012 and will be known as MeerKAT (meer is Afrikaans for more and of course meerkats are rather attractive small mammals that live in the Karoo - see insert to Fig. 4).

If the SKA itself goes to SA the central compact array will be located near Carnarvon, in the Northern Cape, which is an area with particularly low radio interference and will, in future, be protected by legislation (see section 5.1). The exact disposition of the other dishes has yet to be settled, but spreading them throughout Africa, as far east as Mauritius and as far north-west as Ghana, is quite possible.

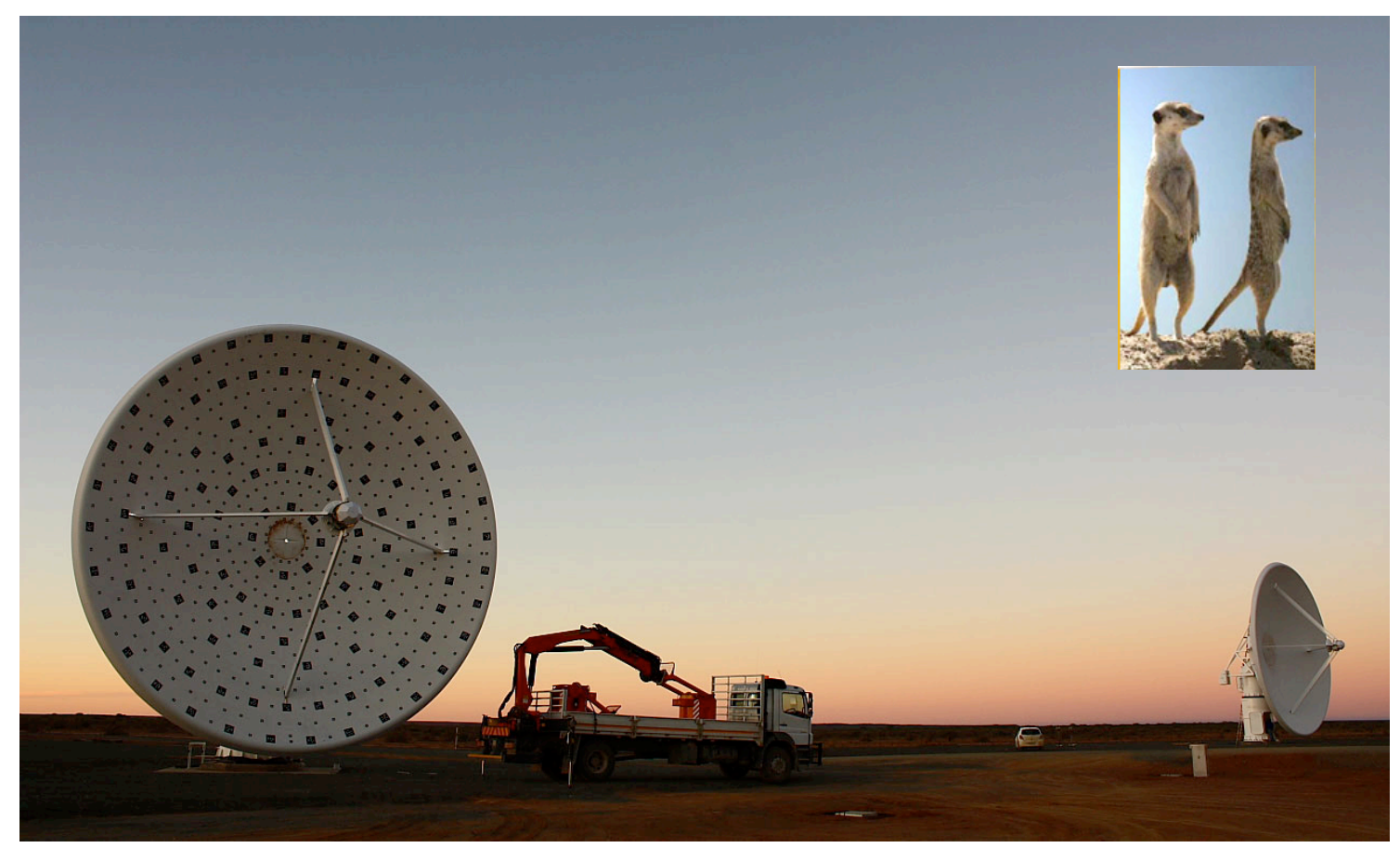

Figure 4 The first two MeerKAT antennae, under construction in the Karoo (insert: meerkats).

\section{Theory and other wavelengths}

South Africa, through the North West University, also participates in the highly successful High Energy Stereoscopic System (HESS) gamma-ray observatory based in Namibia. Although South Africa is only a small player in this field it gives us access to an important wavelength range and provides an opportunity for scientific collaboration with one of our neighbouring countries.

\footnotetext{
${ }^{4}$ www.ska.ac.za
} 
There have never been many people working on theoretical astronomy in South Africa, although George Ellis and his group at the University of Cape Town have certainly made significant contributions to theoretical cosmology. The advent of large telescopes, such as SALT and MeerKAT offer real possibilities for interactions between theorists and observers and it is therefore very exciting to see the formation of a Research Centre in Astrophysics, Cosmology and Gravitation at UCT which will facilitate collaborations and interactions between observers and theorists in cosmology. More broadly it is vital that the South African observing community strengthen their relations with theorists outside of the country.

South Africa is also developing a Virtual Observatory (VO) capability, both to assist with the dissemination of SALT data and to give the local community access to the vast and ever increasing international databases, as well as VO-compatible software/tools for data analysis that will play a vital role in all aspects of astronomy for years to come. This is being done in close collaboration with the Indian Virtual Observatory (VO-I) and with the Inter University Centre for Astronomy and Astrophysics (IUCAA) - who are partners in SALT.

\section{Legislation and Political Developments}

The various political milestones in terms of science policy, vision and legislation were recently described in [2], as were the arguments that led South Africa first to construct SALT and later MeerKAT - despite the serious national challenges of poverty, health and education; the details will not be reiterated here.

It is, however, worth pointing out that discussion and policy documents of South Africa's first democratically elected government acknowledged that we did not have the resources to pursue all scientific endeavours, and could not afford to chose those we did support in an arbitrary way; we must therefore concentrate on those areas where there were either special needs or where we had some special advantages. In particular the 2002 science research strategy document [3] pointed out that astronomy (together with human palaeontology, biodiversity and Antarctic research) was a scientific area in which the country had a clear geographic advantage, and therefore the potential to make internationally recognized contributions. In the case of astronomy this was largely by virtue of its position (latitude, longitude) and climate, but the existing infrastructure was also viewed in a positive light.

The other aspect of science that was strongly emphasized in policy documents over the last decade is the importance of international collaboration and cooperation in all areas of science. Thus when the government first approved funds for SALT it was on the understanding that construction would not start until matching funds had been sourced from international partners. The nature of astronomy has probably made international collaboration a very natural, and even an easy, process for our community.

Other aspects of astronomy were recognized as advantageous for our developing country. It is particularly worth noting that astronomy's use of state-of-the-art 
technology, e.g. electronics, computing and lightweight materials offered opportunities for the transfer of skills and processes that could be broadly useful to industry.

Last, but not least, was the USA and European experience that astronomy was a wonderful vehicle for creating a general interest in science among young people and nudging them into a broad range of scientific careers. This aspect has been a little more problematic in South Africa than first world scientists might have anticipated; there is no doubt that when one is dealing with people who live in real poverty it is difficult to generate much interest in an area which does not obviously offer a lucrative career.

\subsection{Astronomy Geographic Advantages Act}

In 2007 the South African government passed legislation that will protect astronomical sites in the Northern Cape from electromagnetic pollution. This was driven by the need to very clearly protect the potential site for the central part of the SKA. It is, however, an intelligent, well thought out and holistic piece of legislation that should also provide protection to the SAAO at Sutherland. Overall the legislation is clearly aimed at ensuring that South Africa can participate in global scale science projects within partnerships where we provide a good and well-protected location while international partners provide technical and financial resources.

For the optical observatory the main threat is likely to come from mining and particularly from mining for radioactive materials to fuel the country's nuclear energy programme. Nuclear power is also an imperative for the country and even for the Department of Science and Technology - so it is not clear what will happen if a real conflict arises.

For radio astronomy, with so many potential sources of serious interference, the situation is obviously more complex and will require a significant level of policing. Both the radio and optical astronomy communities are aware that the best chance of success is to have the local community on their side, so a great deal of effort is going into educating and informing the community about positive aspects of having an observatory in their area.

\subsection{Space Agency}

In December 2008 the State President signed the Space Agency Act [4] into law and the Agency itself was established in late 2009. This forms part of the DST's 10 year innovation plan [5] as released in 2008, which identifies four "Grand Challenges":

1. Farmer to pharma

2. Space Science

3. Energy

4. Human and Social Sciences

All of the documentation related to the Space Agency suggests that DST views astronomy as part of "Space Science", and early discussions made it clear that they were considering moving the astronomy National Facilities (SAAO, HartRAO and eventually 
MeerKAT) into the Space Agency. Indeed, the Department of Trade and Industry document articulating space policy [6] contains numerous pictures of astronomical objects and a front-cover illustration that includes SALT, the HartRAO 26m antenna and the small telescope domes at Sutherland. Furthermore, the Space Secretariat, which was responsible for many of the preparations for the establishment of the Agency is actually run from SAAO. There are obvious pros and cons to moving astronomy away from the National Research Foundation and into the Space Agency, which has not yet actually happened, and about which the community appears ambivalent. Perhaps because of this, at present the space programme is largely focussed on technical and developmental issues, and the scientific aspects of the space programme are not yet well defined.

\section{Public Relations and Outreach}

There is increasing activity in astronomy, and other scientific, outreach in South Africa. There are also major efforts to coordinate the outreach around the country and to share resources; many of these activities are coordinated through the South African Agency for Science and Technology Advancement (SAASTA), an agency of the NRF financed by DST. The opportunities offered by 2009 as the International Year of Astronomy were really exploited as far as they possibly could be given the resources available - not only in South Africa, but also into many neighbouring countries [7].

Before the construction of SALT was started there was consensus that it must be more than a research tool and that is should provide distinct and substantial benefits in the development of people, technology and the economy. A SALT "collateral benefits" plan was produced; which remains at the heart of many SAAO activities. This identifies the following areas in which SALT could make an impact:

-Educational empowerment (tertiary sector)

-Public outreach

- Direct educational benefits (educators and learners)

-Science education visitor centres

-SALT as an African Facility

It was and is seen as essential that SALT make a major impact on society via the opportunities it presents for a new generation of South Africans to transcend the inequalities of the past and leap over the problems of today.

\section{Growing the Professional Astronomy Community}

In 2009 South Africa had approximately $70 \mathrm{PhD}$ astronomers, almost twice the size of the community in 1998, but still very small by international standards. These scientists are distributed among 12 institutions around the country, with the only critical mass in Cape Town (23 at SAAO, 15 at the University of Cape Town, 5 at the University of the Western Cape and 3 at the Cape Town MeerKAT office). 
While there have been a number of success stories as I outline below, human resource development has been the biggest failure of the system to date and South Africa desperately needs an integrated policy in this area. Several attempts to establish a policy and long term plan for the next decade have simply disappeared within the DST and led to nothing. Significant funding has been devoted to developing the radio astronomy community and given the minute size of the radio community 10 years ago that has been highly successful. The failure to facilitate commensurate developments in optical, high energy and theoretical astronomy is short-sighted and extremely disappointing.

\subsection{The National Astrophysics and Space Science Programme (NASSP)}

It became clear during strategic planning sessions in 2001/2 that the biggest single threat to the future of astronomy in South Africa was the very small numbers of South Africa astronomers and minute numbers of black astronomers. In response to this challenge the astronomers, rapidly joined by the space physics community, set up the National Astrophysics and Space Science programme (NASSP) ${ }^{5}$ [8]. This started in 2003 and takes students with a BSc in physics, applied mathematics or related subject through a one year honours and eighteen-month masters programme so as to prepare them to start on a PhD.

NASSP is a collaboration of 9 South African Universities and 3 National Facilities, based at the University of Cape Town. Students come from all over South Africa and elsewhere in Africa. Since 2009 there has also been an involvement of African American students and lecturers, financed by the Kellogg Foundation through the USA National Society of Black Physicists (NSBP). In addition, a few self-funded European students have participated in NASSP adding to the diversity and strength of the programme.

82 students registered for the 12-month Honours programme between 2003 and 2008, 75 of these graduated 3 withdrew and 4 failed. Of those who graduated 24 are women, 13 are black South Africans, 33 are black Africans and 27 are white South Africans.

48 students registered for the 18-month Masters programme between 2003 and 2008. Of these 25 have graduated, 5 submitted, 1 withdrew, 2 have upgraded to $\mathrm{PhD}$ and 15 are finishing off their projects. Of the 30 students graduated or submitted since 2003, 12 are women, 6 are black South Africans, 10 are black other Africans, 13 are white South Africans and these is one European. 15 graduates are currently studying for a $\mathrm{PhD}$.

\footnotetext{
${ }^{5}$ www.star.ac.za
} 


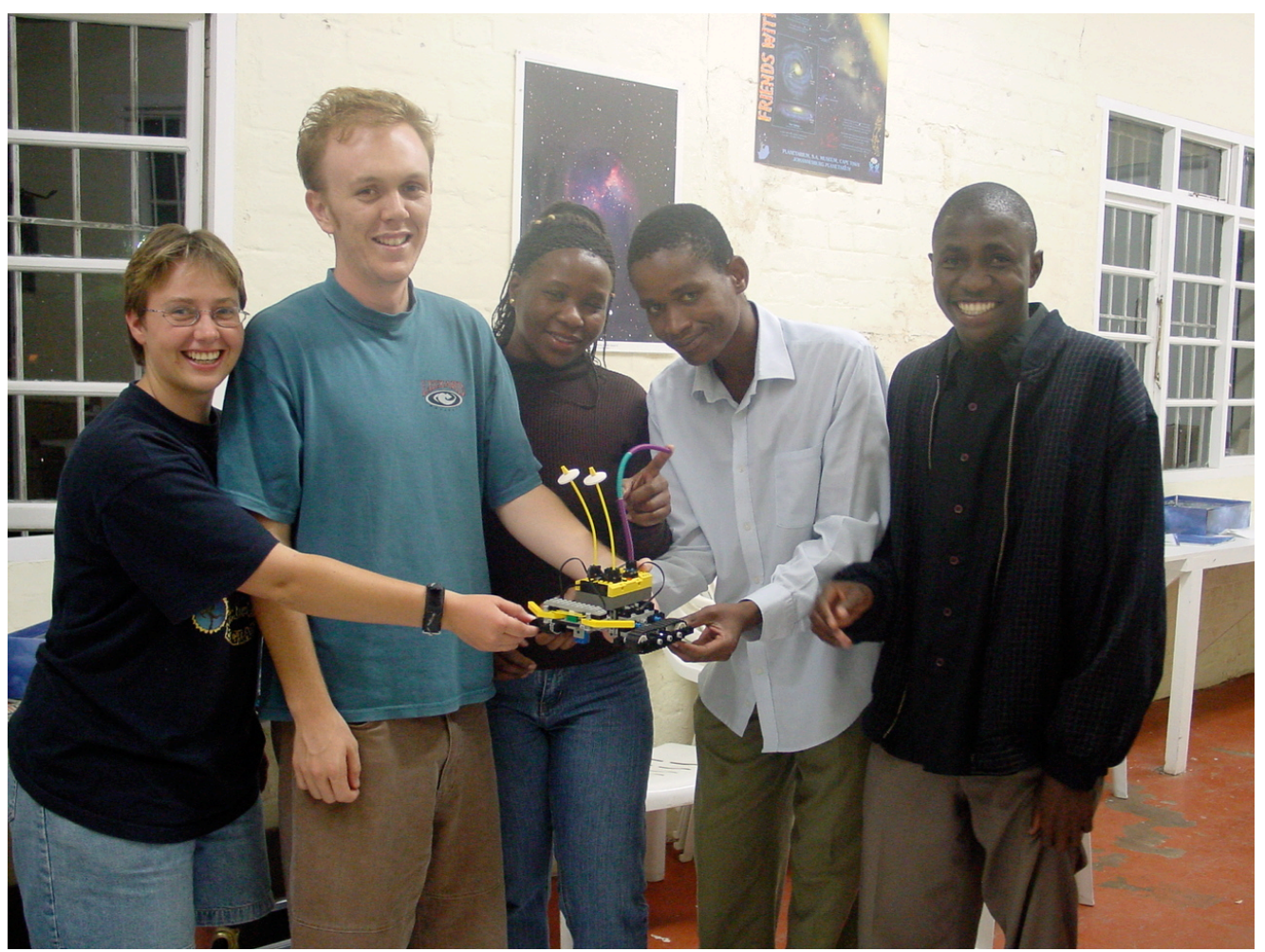

Figure 5 Students at the SAAO NASSP preschool with a robot they have constructed and programmed

NASSP's successes are:

1. Getting more students into astronomy and thereby raising the profile of astronomy both within South Africa and beyond its borders.

2. Getting significant numbers of students into $\mathrm{PhD}$ programmes. Prior to 2003 when NASSP started there was only one student every year or two starting on any $\mathrm{PhD}$ programme in astronomy.

3. Graduating women: 27 percent of the graduates have been women, including some of the top students. Obviously we aim for more, but this is better than any of the other mathematical or physical sciences in South Africa.

4. Graduating students from elsewhere in Africa and starting the process of growing a real community of African astronomers, interlinked professionally and personally. To date students have come from Botswana, Ethiopia, Gabon, Kenya, Madagascar, Mozambique, Rwanda, Sudan, Uganda, Zambia and Zimbabwe. It is no longer necessary to advertise NASSP outside of South Africa and applications far exceed the number of places.

5. Getting the entire South African community to work together. This has not been easy and it is greatly to the credit of the astronomers from outside of the University of Cape Town for making it happen. They were often subjected to severe criticism from their own universities for sending students to UCT. Most of them are now getting MSc and $\mathrm{PhD}$ graduates from NASSP, but it has taken time and patience. 
Its most serious failures are:

1. Attracting too few black South Africans into NASSP.

2. Graduating insufficient black South Africans from NASSP - a large fraction of those who failed or dropped-out of the programme were black South Africans.

These are multiple reasons for this, but the legacy of the Apartheid education system, which the democratic government has hardly impacted upon, is probably the most important. In 2004 fewer than $0.5 \%$ of the black children left school with qualifications that would allow them to enter university to study physics or related subjects [9]; there has been insignificant improvement in the last five years. Most of these qualified students actually study actuarial science, medicine or engineering where the rewards are career paths are clear.

\subsubsection{NASSP Extended Honours Programme}

In response to the major problems highlighted above NASSP started an extended honours programme (EHP) in 2008, with the aim of getting black South African students, not simply to enter NASSP, but do really well once there. This is a pilot programme funded by the Department of Science \& Technology for 3 years. It takes only previously disadvantaged South Africans, i.e. black students who have attended one of the historically disadvantaged universities or who obtained low level passes from a more advantaged institution. Recruitment is done via a winter school held at SAAO that gives the students an opportunity to see what astronomy actually involves and the staff an opportunity to judge the students' commitment and their areas of weakness. Courses cover physics, mathematics, computational methods, English language and introductory astronomy. Most importantly they involve strong support and tutorial assistance for small groups of students (2008: 4; 2009: 7; 2010: 10). The participation of African Americans lecturers in the EHP has been important in demonstrating to the students that it is possible for black people to succeed despite backgrounds of poverty and disadvantage.

The success of this programme is key to the future of NASSP as it is only if this works that the South African government will continue its support. To date it is going well, but it is early days in terms of getting people though $\mathrm{PhDs}$.

\subsubsection{Finance for NASSP}

NASSP is a relatively expensive programme to run and it was clear from the start that it could only work if all of the students received bursaries that covered their fees, travel and living costs. This is indeed the way it was implemented.

NASSP was initially made possible because of the generosity of the Ford Foundation that believed in it when few people thought it could work. Funding for the first few years was from Ford, NRF, UCT, SAAO and HartRAO. Soon after the start contributions from the Canon Collins Trust and the Mellon Foundation allowed for expansion and progress. In recent years the programme has been funded largely from DST, with contributions from NRF, SAAO, the SA-SKA programme and UCT. 
For the future it is clear that DST are not keen to go on funding students from elsewhere in Africa. Those of us involved in the programme believe that broad African involvement is vital for many reasons including South Africa's bid to host the SKA with antennae spread over much of Africa and the fact that the S in SALT stands for Southern, not South. We see South Africa's astronomy future linked to Africa and it is vital that the communities are linked and support each other. We will therefore be approaching donor organizations for help in keeping this link among the African astronomers of the future.

\section{Conclusion}

South Africa stands at the interface between astronomy and development; the challenges are huge, but the potential rewards are even greater. The small South African astronomy community, together with international partners, has made a start on many fronts. Some of these will be very successful, others less so, but what has been achieved in the last 10 to 15 years is actually remarkable: SALT being commissioned; MeerKAT under construction; a successful collaborative postgraduate training programme that is actually empowering some of the most disadvantaged members of the community and an outreach programme that stretches into Africa. The long-term success of each and every one of these projects depends on two factors: local commitment and international involvement. South Africa must drive these projects, but we do not have the skills or the resources to go it alone.

Clearly the IAU's strategic plan [10] with its emphasis on development and Africa is very exciting and is something that South Africa will engage with wholeheartedly. It is, however, vital that we all keep in mind that real success will depend on the level of scientific success of projects such as SALT and MeerKAT, and the extent to which Africans participate in the process of research and discovery.

\section{Acknowledgements}

NASSP is grateful to following organizations for their financial support Ford Foundation, Department of Science and Technology, Mellon Foundation, National Research Foundation, Canon Collins Trust, University of Cape Town, South African Astronomical Observatory and SKA South Africa. NASSP is run from UCT and Directed by Peter Dunsby, but it works because the astrophysics and space physics community want it work - and am very grateful to Peter and to all my colleagues around South Africa and to those from the USA National Society of Black Physicists for making the impossible happen. Finally, thanks to John Menzies for constructive criticism of a draft of this paper.

\section{References}

[1] P. A. Whitelock, M. Dennefeld, B. Leibundgut, 2005, The Scientific Requirements for Extremely Large Telescopes, IAU Sym 232, CUP 
[2] P.A. Whitelock, 2009, Astronomy in Post-Apartheid South Africa, in IAU Sym 260, (eds.) D. VallsGabaud \& A. Boksenberg, The role of Astronomy in Society and Culture, CUP

[3] Department of Science and Technology, 2002, South Africa's Research and Development Strategy, http://www.dst.gov.za/publications-policies/strategies-reports/strategies-reports

[4] South African National Space Agency Act 36 of 2008

[5] Department of Science and Technology, 2008, 10 year Innovation plan, http://www.dst.gov.za/publications-policies/strategies-reports/strategies-reports

[6] Department of Trade and Industry, 2009, South Africa's National Space Policy, http://www.space.gov.za/downloads/Nat Space Policy.pdf

[7] K. Govender, 2009, Astronomy for African Development, in IAU Sym 260, (eds.) D. Valls-Gabaud $\&$ A. Boksenberg, The role of Astronomy in Society and Culture, CUP

[8] P. A. Whitelock, 2008, The South African National Astrophysics and Space Science Programme (NASSP), in (eds.) A. Hady, M. I. Wanas, First Middle East and African IAU-Regional Meeting. http://www.mearim.cu.edu.eg/

[9] OECD, 2007, OECD Reviews of Innovation Policy: South Africa 2007, OECD Publishing

[10] IAU, IAU Strategic Plan 2010-2020, 2009, http://www.iau.org/education/strategic_plan/ 